\title{
Velocidade crítica em natação: uma revisão da literatura
}

\section{Critical velocity in swimming: a literature review}

\author{
Aldo Matos da Costa1, 2, 3*, Mário Jorge Costa2, 4, Daniel Almeida Marinho', 2

\begin{abstract}
De entre as técnicas não invasivas de determinação do limiar anaeróbio, o método da determinação da velocidade crítica tem merecido grande atenção, nomeadamente no âmbito da avaliação e controlo do treino em natação. Neste sentido, o objetivo do presente artigo foi efetuar uma revisão da literatura do tipo qualitativa acerca do significado do parâmetro velocidade crítica e sua aplicação à natação. Foram abordadas questões relativas à origem e definição do conceito de velocidade crítica, às metodologias de determinação mais comuns, bem como uma breve referência ao conceito de velocidade crítica anaeróbia. A velocidade crítica é sugerida como um parâmetro não invasivo de avaliação do desempenho aeróbio e de predição do limiar anaeróbio de nadadores, independentemente do nível desportivo. As principais vantagens deste indicador resultam da facilidade de aplicação e análise num elevado número de nadadores, sem a necessidade da utilização de equipamentos dispendiosos ou de recolha de sangue. Aponta-se como uma ferramenta útil no mecanismo de prescrição e controlo de cargas de treino por meio de resultados em condições experimentais ou recorrendo a tempos obtidos durante as competições. A descriminação dos valores de velocidade crítica entre nadadores com diferentes perfis metabólicos (velocistas versus fundistas) e ainda a sua aplicabilidade em regime anaeróbio são aspetos que ainda carecem de um maior entendimento. Palavras-chave: Avaliação, Desempenho, Nadadores, Treino.
\end{abstract}

ABSTRACT

Considering several non-invasive techniques to determine anaerobic threshold, the determination of critical velocity has been the topic of interest of several studies, namely under the scope of evaluation and control of training in swimming. Therefore, the aim of this study was to conduct a literature review regarding the meaning of the critical velocity and its application in swimming. The concept origins, the critical velocity definition and the most common methodologies for its determination were addressed. The critical velocity has been known as a non-invasive measure that represents the aerobic capacity and is similar to anaerobic threshold in swimmers, independent from the competitive level. Major advantages are the easily application in field interventions without having to use heavy methods/blood samples collection, and the possibility to comprise larger samples of swimmers for further analysis. The critical velocity is a useful tool for training load prescription and seasonal monitorization that can be assessed by mean of experimental approach or using race times in competition context. The discrimination about critical velocity values regarding swimmers with different metabolic profiles and the use of the concept within the anaerobic context are aspects that need further understanding.

Keywords: evaluation, performance, swimmers, training.

Artigo recebido a 26-08-2013; Aceite a 22-02-2015

${ }^{1}$ Universidade da Beira Interior, Departamento de Ciências do Desporto, Covilhã, Portugal

${ }^{2}$ Centro de Investigação em Desporto, Saúde e Desenvolvimento Humano (CIDESD), Portugal

${ }^{3}$ Centro de Investigação em Ciências da Saúde (CICS-UBI), Portugal

${ }^{4}$ Instituto Politécnico da Guarda, Escola Superior de Educação, Comunicação e Desporto, Guarda, Portugal

* Autor correspondente: Universidade da Beira Interior, Rua Marquês D’Ávila e Bolama, 6200-001 Covilhã; E-mail: amcosta@ubi.pt 


\section{INTRODUÇÃO}

Assistimos nas comunidades técnica e científica à emergência de um forte interesse em desenvolver critérios, testes e métodos de avaliação com diferentes vocações e incidências, diferentes níveis de complexidade e potencial de retroacção. Nesse sentido, ao longo dos anos, o volume máximo de oxigénio $\left(\mathrm{VO}_{2 \max }\right)$ tem sido considerado o parâmetro ex-libris para a avaliação da potência aeróbia em indivíduos ativos, sedentários e atletas, com base na sua importante relação com o desempenho, particularmente em esforços físicos intensos e de duração inferior a 10 minutos (Jones \& Poole, 2005).

Na natação, particularmente nos eventos mais longos, a literatura parece declinar a existência de uma relação robusta entre $\mathrm{o} \quad \mathrm{VO}_{2 \max } \mathrm{e} \quad \mathrm{o}$ desempenho de nado (Fernandes \& Vilas-Boas, 2012; Reis, Marinho, Policarpo, Carneiro, et al., 2010; Reis, Marinho, Policarpo, Reis, et al., 2010). Assim, e adicionando as limitações impostas pela estimação e utilização do $\mathrm{VO}_{2 \max }$ em treino, a resposta do lactato sanguíneo ao exercício tem sido amplamente empregue, a partir da determinação do limiar anaeróbio com base na curva de lactatemia vs. velocidade de nado (Billat, Sirvent, Py, Koralsztein, \& Mercier, 2003; Fernandes \& Vilas-Boas, 2012; Mader \& Heck, 1986). Este indicador é considerado um referencial para a prescrição da intensidade de treino, controlo dos seus efeitos e predição do desempenho aeróbio.

Para a determinação do limiar anaeróbio existem diferentes metodologias que se baseiam em protocolos que medem de forma direta a concentração de lactato sanguíneo, utilizando concentrações fixas (Heck et al., 1985) ou individualizadas (Stegmann, Kindermann, \& Schnabel, 1981). Realça-se, entretanto, a metodologia de Tegtbur, Busse, e Braumann (1993) que converteu para a natação a metodologia de lactato mínimo, permitindo a identificação do máximo estado estável de lactato de maneira individualizada, com aplicação em inúmeros estudos em natação (Fernandes, Sousa, Machado, \& Vilas-Boas, 2011; Ribeiro, Balikian, Malachias, \& Baldissera, 2003).
Apesar das novas facilidades tecnológicas que permitirem resultados em poucos segundos, os testes de lactatémia assentam em técnicas invasivas com custos significativos em materiais descartáveis. Por isso temos assistido à tentativa de desenvolver outros métodos e critérios alternativos. Como o conceito potência crítica foi aplicado com bastante eficácia ao esforço físico cíclico (ciclo ergómetro) (Moritani, Nagata, deVries, \& Muro, 1981), o seu transfere e adaptação à natação proposto por Ettema (1966) veio a tornar-se uma mais-valia na avaliação e inclusive prescrição do treino, dando origem aquilo que é hoje conhecido como velocidade critica (VC) (Wakayoshi, Ikuta, et al., 1992; Wakayoshi, Yoshida, et al., 1992). A maioria dos estudos na natação determina a VC com base na regressão linear entre distancias fixas e os respectivos tempos, correspondendo ao coeficiente angular da recta obtida. Contudo, a variabilidade das coordenadas e distâncias utilizadas é grande e algumas das pesquisas incluem eventos com duração inferior a 2 minutos, procedimento não recomendado dado que o esforço não possibilita que o $\mathrm{VO}_{2 \max }$ seja atingido (Dekerle, Brickley, Sidney, \& Pelayo, 2006). O recurso a distâncias mais longas parece subestimar a VC enquanto que a inclusão de distâncias mais curtas parece conduzir a uma sobreestimação. Esta varibilidade metodológica parece ter induzido alguma divergência conceptual acerca deste indicador, dado que diferentes valores de VC são obtidos sobre tempos de exaustão variados (Zacca et al., 2010), para além da influência nem sempre balizada de vários aspectos relativos às caracteristicas dos nadadores, em particular a idade e a experiência desportiva (Franken, Zacca, \& Castro, 2010).

Adicionalmente, o modelo com 2 parâmetros (i.e a partir da relação hiperbólica entre a potência e o tempo para a exaustão) tem sido questionado por vários autores dado que parece sobrestimar a potência crítica e subestimar a capacidade de trabalho anaeróbia (a interceção na origem desta reta) (eg. di Prampero, 1999; Morton, 1996, 2006; Vandewalle, Kapitaniak, Grün, Raveneau, \& Monod, 1989; Zacca et al., 2010). Assim, novos modelos bioenergéticos de 3 e 4 parâmetros têm procurado atenuar estas limitações 
com importantes implicações para o significado fisiológico da VC.

A maioria dos eventos desportivos em natação são iguais ou inferiores a $200 \mathrm{~m}$, pelo que o rendimento desportivo está muito dependente do metabolismo anaeróbio do atleta (Housh et al., 1991; Smith \& Hill, 1993). Desse modo, a VC em regime de nado anaeróbio $\left(\mathrm{VC}_{\mathrm{an}}\right)$ será de especial interesse na avaliação da aptidão anaeróbia dos nadadores (di Prampero, Dekerle, Capelli, \& Zamparo, 2008; Marinho, Barbosa, Silva, \& Neiva, 2012; Neiva, Fernandes, \& Vilas-Boas, 2011). Nos últimos anos alguns estudos têm sido publicados sobre a relevância deste indicador enquanto parâmetro de avaliação e controlo do treino anaeróbio do nadador e sua relação com o desempenho desportivo em eventos competitivos de curta duração.

Assim, foi nosso objectivo efetuar uma revisão da literatura do tipo narrativa acerca do significado do parâmetro VC e sua aplicação à natação. $\mathrm{O}$ parâmetro emergente de $\mathrm{VC}_{\mathrm{an}}$ foi igualmente alvo de discussão. O âmbito deste trabalho conduziu-nos a uma pesquisa de carácter qualitivo compreensivo-interpretativo, recorrendo a palavras-chave na língua Portuguesa e Inglesa (natação, nadadores, velocidade critica, velocidade critica anaeróbia) nas bases de dados Index Medicus, MEDLINE, Science. Adicionalmente estendeu-se a pesquisa aos nossos ficheiros de departamento, incluindo livros de encontros científicos (p.e. Biomechanics and Medicine in Swimming, European College of Sports Science, International Symposium on Biomechanics in Sports) para um maior alcance da revisão efetuada. Deste modo foram seleccionados 66 trabalhos entre 1965 (ano no qual o conceito de potência crítica foi introduzido por Monod e Scherer) e Maio de 2014.

\section{Origem e definição do conceito}

O conceito de VC sugerido por Ettema (1966) e aplicado pela primeira vez por Wakayoshi et al. (1992), deriva do conceito de potência critica $\left(\mathrm{PC}_{\text {rit }}\right)$ originalmente introduzido por Monod e Scherer (1965), procurando entender a capacidade de trabalho local de um músculo ou de grupos musculares sinérgicos. Estes autores sugeriram que o trabalho total local realizado antes da exaustão durante um exercício supramaximal de intensidades crescentes poderia ser descrito como a soma das quantidades de trabalho produzido com base no metabolismo anaeróbio e aeróbio (di Prampero, 1999). Assim, para exercícios dinâmicos, a $\mathrm{PC}_{\text {rit }}$ define-se como a intensidade de exercício que pode ser mantida sem exaustão (teoricamente), sendo determinada pelo declive da recta de regressão entre o trabalho total e o tempo correspondente até à exaustão (Hill, Steward Jar, \& Lane, 1995).

Mais tarde, por Moritani et al. (1981), o conceito de $\mathrm{PC}_{\text {rit }}$ veio a ser aplicado ao trabalho corporal total realizado em cicloergómetro. Estes autores observaram que a $\mathrm{PC}_{\text {rit }}$ representava um índice de desempenho aeróbio, relacionada significativamente com o $\mathrm{VO}_{2 \max }\left(\mathrm{ml} . \mathrm{kg} \cdot \mathrm{min}^{-1}\right)(\mathrm{r}$ $=0.919)$ e com o limiar anaeróbio ventilatório $(\mathrm{r}$ $=0.927)$. Teoricamente a $\mathrm{PC}_{\text {rit }}$ representa $\mathrm{a}$ maior potência que se pode manter por tempo indefinido em estado de equilíbrio fisiológico aeróbio (Hill, 1993; Hill et al., 1995), demarcando a transição entre o domínio da intensidade de esforço elevada e a severa (Brickley, Doust, \& Williams, 2002; Poole, Ward, Gardner, \& Whipp, 1988).

Este conceito, baseado na relação linear trabalho-tempo ou potencia-tempo ou ainda, alternativamente, na relação hiperbólica potencia-tempo, engloba a contribuição aeróbia e anaeróbia para a totalidade da potencia produzida por unidade de tempo. Assim, para gestos cíclicos onde a distância e a velocidade são geridas de acordo com o tempo de esforço, a expressão "velocidade crítica" é utilizada de forma mais comum alternativamente à "potência crítica" (Berthoin, Baquet, Dupont, \& Van Praagh, 2006), a qual é dada pela inclinação da linha de regressão entre distância percorrida $\left(d_{\text {lim }}\right)$ e o tempo até a exaustão $\left(t_{\text {lim }}\right)$, representando a interceção em y a capacidade anaeróbia do atleta (Ettema, 1966):

$$
d_{\mathrm{lim}}=\left(\mathrm{VC} \cdot t_{\mathrm{lim}}\right)+\mathrm{CCA}
$$




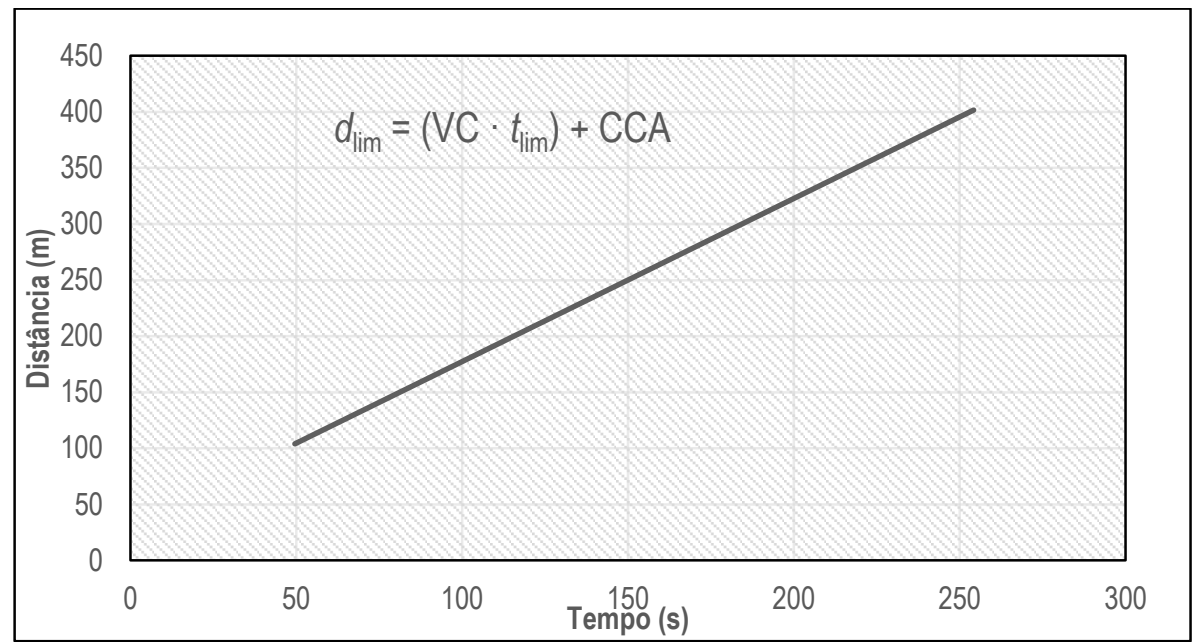

Figura 1. Relação linear entre a distância percorrida e o tempo até a exaustão, originalmente proposta por Ettema (1966).

A proposta de reconversão do modelo de $\mathrm{PC}_{\text {rit }}$ para a especificidade da natação foi inicialmente sugerida por Ettema (1966), tendo sido Wakayoshi et al. (1993; 1992a,b) os primeiros a efectivamente aplicarem o conceito. Nesse primeiro estudo Wakayoshi et al. (1992a) recorreram a seis velocidades de nado para o cálculo do coeficiente angular entre $d_{\text {lim }}$ e $t_{\text {lim }}\left(\mathrm{r}^{2}>\right.$ 0,998), aplicando o conceito de VC de nado, significativamente correlacionada com $\mathrm{O} \quad \mathrm{VO}_{2}$ correspondente ao limiar anaeróbio $(r=0.818$, $\mathrm{p}<0.01$ ), com a velocidade de nado no início da acumulação de concentração de ácido lactico $(\mathrm{r}=$ 0.949, $\mathrm{p}<0.01)$ e com a velocidade de nado média nos $400 \mathrm{~m}$ livres $(\mathrm{r}=0,865, \mathrm{p}<0.01)$. Assim, na perspectiva dos autores, a VC (neste caso particular obtida com recurso a seis coordenadas) corresponderia à velocidade de nado que teoricamente pode ser mantida por um período mais prolongado de tempo sem exaustão. Foi, portanto, considerada em vários estudos como um índice de grande potencial, cujo rigor e natureza não invasiva permite uma avaliação simplificada, embora criteriosa, do desempenho aeróbio e de predição do limiar anaeróbio de nadadores adultos (Rodriguez, Moreno, \& Keskinen, 2003; Wakayoshi, et al., 1993; 1992a,b). Recentemente, outros autores confirmaram a aplicabilidade do conceito em nadadores jovens (Hill et al., 1995), de ambos os géneros (Costa et al., 2009; Greco \& Denadai, 2005) e independentemente do seu nível desportivo (Denadai, Greco, \& Teixeira, 2000). Tal como refere
Vilas-Boas, Lamares, Fernandes e Duarte (1997), parece tratar-se de um parâmetro facilmente associável à ideia de intensidade máxima aeróbia, imediatamente inferior à que provocaria um desequilíbrio entre a produção e a remoção ou a utilizacão de lactato sanguíneo, ou seja, esperadamente correlacionado com a velocidade em torno do limiar anaeróbio (Greco, Denadai, Pellegrinotti, Freitas, \& Gomide, 2003; Wakayoshi et al., 1992b; Wright \& Smith, 1994) e com o steady state máximo de lactato (Wakayoshi et al., 1993). Não obstante o aspecto prático deste indicador, o seu significado fisiológico é ainda inconclusivo pois está dependente sobretudo do modelo de cálculo e das diferentes combinações de distâncias e coordenadas utilizadas.

\section{Modelos, combinações de distâncias e coorde- nadas mais comuns para o cálculo da VC em na- tação}

A VC tem sido determinada de várias formas (tabela 1), destacando-se o recurso ao modelo de 2 parâmetros, quer utilizando diretamente a regressão distância/tempo, quer utilizando uma equação de regressão previamente calculada entre a velocidade num teste de determinada distância e a VC (Wakayoshi et al., 1993; 1992a,b; Wright \& Smith, 1994).

De facto, ao longo dos anos foram vários os autores que se suportaram nesta metodologia recorrendo aos melhores tempos dos nadadores em condições experimentais (simuladas) e de prova. Wakayoshi et al. (1993) e Dekerle, Sidney, 


\section{2 | AM Costa, MJ Costa, DA Marinho}

Tabela 1.

Alguns estudos centrados no cálculo da velocidade crítica em natação com base na combinação de coordenadas e distâncias de nado.

\begin{tabular}{|c|c|c|c|c|c|}
\hline \multirow{2}{*}{ Referência } & \multicolumn{2}{|l|}{ Amostra } & \multirow{2}{*}{ Distâncias de nado (m) } & \multirow{2}{*}{ Modelo } & \multirow{2}{*}{ Resultados } \\
\hline & $\mathrm{N}$ & Idade (anos) & & & \\
\hline Wakayoshi et al. (1992a) & 9 nadadores, universitários & $(18-21)$ & $\begin{array}{l}6 \text { velocidades pré-determina- } \\
\text { das }\end{array}$ & Linear, 2 parâmetros & $\begin{array}{l}\text { Correlação positiva e significativa com o consumo de oxigénio } \\
\text { no limiar anaeróbio }(\mathrm{r}=0,818), \mathrm{V}_{\text {OBLA }}(\mathrm{r}=0.94) \text { e com a veloci- } \\
\text { dade média dos } 400 \mathrm{~m}(\mathrm{r}=0.864) \text {. }\end{array}$ \\
\hline Wakayoshi et al. (1993) & 8 nadadores, universitários & $(18-21)$ & 200 e 400 & Linear, 2 parâmetros & $\begin{array}{l}\text { Correlação significativa com a velocidade do limiar anaeróbio } \\
(\mathrm{r}=0.914) \text { e com a velocidade dos } 400 \mathrm{~m} \text { crol }(\mathrm{r}=0.977)\end{array}$ \\
\hline Wright \& Smith (1994) & $\begin{array}{l}19 \text { nadadores, nível nacional } \\
\text { (12 homens e } 7 \text { mulheres) }\end{array}$ & $(>18)$ & $\begin{array}{l}50-200-600 \\
\text { e 1200; } \\
50-200-600\end{array}$ & Linear, 2 parâmetros & $\begin{array}{l}\mathrm{VC}_{50 / 200 / 600} \text { significativamente diferente }\left(\begin{array}{ll}\mathrm{p} & <0.05) \\
\mathrm{VC}_{50 / 200 / 600 / 1200}\end{array}\right. \\
\text { Correlação positiva e significa entre velocidade dos } 1200 \mathrm{~m} \text { e a } \\
\mathrm{VC}_{50 / 200 / 600 / 1200}(\mathrm{r}=0.99)\end{array}$ \\
\hline Dekerle et al. (2002) & $\begin{array}{l}10 \text { nadadores, treinados } \\
\text { (8 homens e } 2 \text { mulheres) }\end{array}$ & $(18.6 \pm 1.9)$ & 200 e 400 & Linear, 2 parâmetros & $\begin{array}{l}\text { VC similar à velocidade de nado no teste máximo de } 30 \text { minu- } \\
\text { tos (sobrestimando em } 3.2 \% \text { ) }\end{array}$ \\
\hline Greco et al. (2003) & 31 nadadores, treinados & (10 à 15) & $\begin{array}{l}25,50 \text { e } 100 \\
100,200 \text { e } 400 \\
50,100 \text { e } 200\end{array}$ & Linear, 2 parâmetros & $\begin{array}{l}\text { A distância utilizada interfere com a velocidade critica calcu- } \\
\text { lada, independente da idade cronológica; distâncias de } 50 \text { e } \\
400 \mathrm{~m} \text { sugeridas para avaliação da capacidade aeróbia nestas } \\
\text { faixas etárias. }\end{array}$ \\
\hline Papoti et al. (2005) & 8 nadadores, nível nacional & $(17 \pm 0.1)$ & 200 e 400 & Linear, 2 parâmetros & $\begin{array}{l}\text { VC não difere significativamente da velocidade de limiar anae- } \\
\text { róbio; correlação significativa com a performance em } 400 \mathrm{~m} \\
\text { crol }(\mathrm{r}=0.91)\end{array}$ \\
\hline Altimari et al. (2007) & 15 nadadores, nível nacional & $(14.7 \pm 0.7)$ & $\begin{array}{c}50,100 \text { e } 200 \\
100,200 \text { e } 400 \\
50,100,200 \text { e } 400 \\
200 \text { e } 400\end{array}$ & Linear, 2 parâmetros & $\begin{array}{l}\text { Correlação negativa e significativa com a performance (s) nos } \\
400 \mathrm{~m}(\mathrm{r}=-0.62 ; \mathrm{r}=-0.97 ; \mathrm{r}=-0.98 ; \mathrm{r}=-0.94 \text {, respetivamente) }\end{array}$ \\
\hline Zacca \& Castro (2009) & 11 nadadores, nível nacional & $(14.4 \pm 0.5)$ & $\begin{array}{c}\text { 50 e } 200 ; \\
100 \text { e } 400 ; \\
200 \text { e } 800 ; \\
400 \text { e } 1500 ; \\
50,100 \text { e } 200 ; \\
100,200 \text { e } 400 ; \\
200,400 \text { e } 800 ; \\
400,800 \text { e } 1500 ; \\
50,100,200 \text { e } 400 \\
\end{array}$ & Linear, 2 parâmetros & $\begin{array}{l}\text { Distâncias de nado inferiores a } 60 \text { segundos }(50 \text { e } 100 \mathrm{~m}) \text { con- } \\
\text { duzem a valores de VC elevados, pelo que não devem ser usa- } \\
\text { dos na sua estimação. } \\
\text { O uso das distâncias longas ( } 800 \text { e } 1500 \mathrm{~m} \text { ) não altera signifi- } \\
\text { cativamente a VC comparativamente às combinações com dis- } \\
\text { tâncias entre os } 200 \text { e os } 400 \mathrm{~m} \text {. }\end{array}$ \\
\hline Costa et al. (2009) & 24 nadadores, nível nacional & $(15.04 \pm 0.20)$ & 100,200 e 400 & Linear, 2 parâmetros & $\begin{array}{l}\text { VC similar à velocidade de nado de limiar anaeróbio e do teste } \\
\text { de } 30 \text { minutos de nado contínuo }\end{array}$ \\
\hline Zacca et al. (2010) & $\begin{array}{l}14 \text { nadadores, treinados } \\
\text { (7 velocistas e } 7 \text { fundistas) }\end{array}$ & $(14-15)$ & $50,100,200,400,800$ e 1500 & $\begin{array}{l}\text { Linear, } 2 \text { parâmetros } \\
\text { Não linear, } 3 \text { parâmetros } \\
\text { Não linear, } 4 \text { parâmetros }\end{array}$ & $\begin{array}{l}\text { O modelo de } 3 \text { parâmetros parece ser o mais ajustado embora } \\
\text { o modelo de } 4 \text { parâmetros pareça ser mais apropriado para na- } \\
\text { dadores de endurance. }\end{array}$ \\
\hline
\end{tabular}


Hespel, e Pelayo (2002) definiram as distâncias de 200 e $400 \mathrm{~m}$ para a sua determinação, independentemente do contexto de realização.

Wright e Smith (1994) propuseram uma equação para a predição da VC através de um teste de $1200 \mathrm{~m}$, tendo por base uma equação de regressão entre o tempo dos $1200 \mathrm{~m}$ (V1200) e a $\mathrm{VC}(\mathrm{VC}=1,017 \times \mathrm{V} 1200-0,039)$. Mais tarde, Fernandes e Vilas-Boas (1999) apontaram a possibilidade do uso das distâncias de 200 e 800 m como alternativa para a estimativa da VC. Mais recentemente Costa et al. (2009) sugeriram a utilização dos melhores tempos do nadador em situação real de prova nas distâncias de $100 \mathrm{~m}$, $200 \mathrm{~m}$ e $400 \mathrm{~m}$, estimando uma intensidade de nado em VC similar $(\mathrm{p}<0.05)$ à velocidade de nado em torno do limiar anaeróbio.

Os métodos propostos tornaram-se populares pela sua facilidade operacional, cultivando-se como uma ferramenta útil para treinadores no processo de planeamento. Aparte da simplicidade operacional, há que ter em conta que os valores da regressão estão dependentes dos tempos de exaustão usados para o cálculo da relação, e que de certa forma influenciam o custo energético de nado (di Prampero, 1999). Com efeito, alguma controvérsia começou a surgir perante estas abordagens. Alguns autores salientam que os esforços máximos contínuos de duração inferior a 2 minutos não possibilitam que o $\mathrm{VO}_{2 \max }$ seja atingido (Dekerle et al., 2006). Assim, é recomendado para o cálculo da VC incluir distâncias no modelo de regressão que permitam a obtenção e estabilização do $\mathrm{VO}_{2 \max }$ (entre 2 a 15 min) (Dekerle et al., 2006). No caso da natação, as distâncias entre 200 e $1500 \mathrm{~m}$ parecem ser as mais fiáveis embora não pareça ser importante recorrer a distâncias superiores a $400 \mathrm{~m}$ em jovens nadadores (Zacca \& Castro, 2009). Mais ainda, dado que a VC geralmente é estimada com base num modelo de distancia-tempo, recorrendo ao melhor tempo do nadador em distâncias crescentes, o indicador é sensível à orientação desportiva dos nadadores. Assim, será expectável que os nadadores fundistas obtenham valores mais elevados de VC sobretudo quanto maiores forem as distâncias de nado incluídas no modelo. Esta é uma preocupação a reter no desenho de estudos experimentais no futuro, dado que pode atribuir heterogeneidade à amostra selecionada.

Outros autores consideraram que o uso de apenas duas distâncias de nado como referência poderia acarretar alguma subjetividade no cálculo da VC (Dekerle et al., 2006). Esta preocupação assentou na variabilidade de tempos de exaustão tendo em vista a determinação da relação distância-tempo o que poderia, de certa forma, impossibilitar a comparação de resultados científicos e desvirtuar o planeamento do treino. Mesmo assim, parece sustentado que o cálculo da VC seria fiável mesmo com o uso de tempos de exaustão variados (Hinckson \& Hopkins, 2005). No entanto, do ponto de vista teórico, quanto maior for o número de distâncias nadadas incluídas na determinação da VC, maior será o potencial da linha de regressão e os possíveis erros serão minimizados. De acordo com Altimari, Altimari, Gulak, e Chacon-Mikahil (2007) o número de eventos para a estimativa da VC (e por inerência, o número de sessões de teste) pode ser reduzido em nadadores jovens, desde que utilizadas distâncias que permitem um tempo de esforço até à ocorrência de exaustão.

A literatura tem ainda procurado modificar o modelo de dois parâmetros com vista a melhorar a sua validade dado que este é assente em alguns pressupostos que não parecem ser fisiologicamente realistas. Um desses pressupostos está relacionado com a improbabilidade de existir uma VC instantânea no início do exercício (tempo zero), o que parece conduzir à sua sobrestimação (Gaesser, Carnevale, Garfinkel, Walter, \& Womack, 1995; Morton, 1996, 2006). Procurando corrigir essa falha matemática, Morton (1996) propôs um modelo com um parâmetro adicional representando a velocidade no tempo zero, designada por velocidade máxima instantânea. Isso permitiu estimar uma VC mais baixa e por consequência uma maior sustentabilidade dessa intensidade do esforço (Gaesser et al., 1995). Outro pressuposto fisiologicamente improvável do modelo de dois parâmetros assenta na não predição da "inércia aeróbia", ignorando o ajustamento cardiorrespiratório necessário para a estabilidade do consumo de oxigénio (Vandewalle et al., 1989; Wilkie, 1980). Essa carência foi corrigida por Wilkie (1980) pela introdução 
de uma correção para a cinética do consumo de oxigénio, através de um fator exponencial com constante temporal (inércia aeróbia) que representa o aumento da participação aeróbia. Com vista atenuar as insuficiências dos modelos de 2 e 3 parâmetros,

Num estudo recente no contexto da natação desportiva, Zacca et al. (2010) identificaram diferenças nos valores da VC quando utilizados diferentes modelos (2, 3 e 4 parâmetros), obtidos a partir de seis distâncias e tempos diferentes. Contudo a introdução de um quarto parâmetro (inércio aeróbia) não parece ter trazido uma melhoria qualitativa no ajustamento da VC em comperação com o modelo de três parâmetros. Para além disso, refere o autor, que a estimação da VC por via destes modelos matemáticos mais elaborados (3 e 4 parâmetros) pressupõem o recurso a cinco ou seis esforços máximos, o que lhe retira praticidade.

\section{Evidências científicas da aplicação do conceito de Velocidade Crítica à natação}

Tal como atrás referimos, o conceito original de VC aplicado à natação (Wakayoshi et al., 1993; 1992a,b) é definido como a velocidade máxima de nado teórica que pode ser mantida sem atingir a fadiga por um longo período de tempo. No entanto, a aplicação deste conceito não está livre de controvérsia (Dekerle et al., 2005; Denadai et al., 2000; Martin \& Whyte, 2000). Por isso a delimitacão concreta da intensidade da zona metabólica à qual a VC melhor se associa é uma preocupação fulcral.

Sabemos que as variáveis relacionadas com a cinética do $\mathrm{VO}_{2}$ reflectem a velocidade com que o sistema aeróbio responde, permitindo estimar a contribuicão dos sistemas aeróbio e anaeróbio durante o exercício (Hill, 2001; Reis, Marinho, Policarpo, Carneiro, et al., 2010; Reis, Marinho, Policarpo, Reis, et al., 2010; Spencer \& Gastin, 2001). Segundo Whipp e Wasserman (1972), durante o exercício sub máximo, os sujeitos com a melhor aptidão aeróbia respondem mais rapidamente e apresentam menor défice de oxigénio; enquanto aos indivíduos com menor aptidão aeróbia acontecerá o oposto (Whipp \& Wasserman, 1972).
A VC aplicada à natacão, derivada do modelo de "potencia crítica" de Monod e Scherrer (1965), enquanto parâmetro associado a uma intensidade de esforço em torno do máximo estado estável de $\mathrm{VO}_{2}$ (Hill \& Ferguson, 1999). Aliás, a validade da igualdade entre estes dois indicadores, potência crítica e máximo estado estável de $\mathrm{VO}_{2}$, teria já sido demonstrada por Poole et al. (1988; 1990). Nestes estudos, em exercício prolongado à intensidade da potencia crítica, houve estabilização do $\mathrm{VO}_{2}$ em cerca de $80 \%$ do seu valor máximo. Foi ainda verificado que um incremento de apenas $5 \%$ de carga provocaria um aumento do $\mathrm{VO}_{2}$ até atingir o seu pico máximo, precipitando a exaustão em poucos minutos. Esta sustentação fisiológica veio a ser igualmente proposta por Hill e Smith (1999), com outra técnica de estimação da intensidade associada ao máximo estado estável de $\mathrm{VO}_{2}$, adoptando alguns dos pressupostos do modelo de Monod e Scherrer (1965).

Porém, na natação a relação distância-tempo não parece ser estritamente linear (Dekerle et al., 2002; Wright \& Smith, 1994), dado que custo energético não aumenta exponencialmente quando a velocidade de nado aumenta (Capelli, Pendergast, \& Termin, 1998). Para além disso, parâmetros tais como a força propulsiva e arrasto ativo podem interferir na eficiência propulsiva e por inerência no dispêndio energético (di Prampero et al., 2008; Ettema, 1966; Zamparo, Capelli, \& Pendergast, 2011).

De facto, diversos autores (Brickley et al., 2002; Dekerle et al., 2006) parecem concordar que a VC poderá não corresponder à sustentação de uma intensidade de nado ao longo de um período prolongado, precipitando-se a exaustão com apenas ligeiros incrementos de velocidade. Num estudo relativamente recente de Dekerle, Brickley, Sidney, e Pelayo (2006), os nadadores somente foram capazes de manter a VC durante 30 a 40 minutos. Por sua vez, outros estudos indicam-na como próxima da velocidade de nado num teste máximo de 30 minutos (Dekerle et al., 2002) e ligeiramente superior ao máximo estado estável de lactato e da velocidade de nado média estimada para concentrações de lactato de 4 m.mol-1 (Dekerle et al., 2005; Denadai, Greco, \& Teixeira, 2000; Rodriguez, Moreno, \& Keskinen, 
2003; Wakayoshi et al., 1993; 1992a,b). Adicionalmente, sabemos que a velocidade correspondente a esse estado metabólico está correlacionada negativamente com o tempo limite para a exaustão em velocidade de $\mathrm{VO}_{2 \max }$ (Fernandes et al., 2003). Conhece-se ainda, no que se refere a este último indicador, uma associação igualmente negativa com custo energético de nado à velocidade ao $\mathrm{VO}_{2 \max }$ e com a velocidade de nado efectiva ao $\mathrm{VO}_{2 \max }$ (Fernandes et al., 2006), embora não influencie significativamente no $\mathrm{VO}_{2 \max }$ (Cardoso, Fernandes, \& Vilas-Boas, 2003; Faina et al., 1997; Fernandes et al., 2003). Assim, segundo Whipp e Wasserman (1972), quanto maior o tempo necessário para atingir um estado estável de $\mathrm{VO}_{2}$, maior será a participação anaeróbia para síntese de ATP e prematura a interrupção do esforço devido a fadiga.

Posto isto, o nado à intensidade de VC poderá, também, ter uma considerável participação do metabolismo anaeróbio, embora sempre abaixo de uma intensidade metabólica severa ou submáxima. Corresponderá, portanto, a um domínio de exercício que terá como limite inferior a carga de trabalho na qual o balanço entre produção e remoção de lactato pende transitoriamente para o primeiro e terá, como limite superior, a carga referente à máxima concentração estável de lactato. Segundo Gaesser e Poole (1996), essa intensidade demarca a transição entre o domínio intenso e o severo de esforc,o, patamar este que coincide com a potencia crítica. Deste modo, a VC parece ser um conceito claramente diferente de uma dada velocidade de nado mantida em regime aeróbio de longa duração por nadadores experientes no âmbito da sua percepção interna de intensidade de esforço (Toubekis, Tsami, Smilios, Douda, \& Tokmakidis, 2011).

Recorrendo-se à VC como indicador de aptidão aeróbia podemos avaliar os efeitos da intensidade do exercício no nadador dado que a VC fornece um índice de sustentabilidade máxima da intensidade de nado e que a amplitude da braçada diminui abaixo do limiar anaeróbio (Dekerle et al., 2005; Keskinen \& Komi, 1993). Assim, de acordo com Dekerle et al. (2005), será muito diferente nadar a velocidades superiores à VC do que a velocidades mais baixas. De acordo com a autora, o conceito VC, está associado ao momento em que se dão mudanças substanciais na frequência gestual e na ocorrência da amplitude do ciclo gestual. Para além disso, a relação linear entre a frequência gestual e a velocidade poderá ser usada para predizer a VC, e que esta predição pode ser facilmente obtida a partir da observação da frequência em ritmo normal o ciclo de membros superiores (Barden \& Kell, 2009). Assim, quer a VC quer a frequência gestual crítica (obtida com base na relação linear entre o número de ciclos de membros superiores e o tempo obtido em cada distância) serão indicadores relevantes de avaliação fisiológica e técnica do nadador, permitindo ainda um controlo simultâneo da intensidade e da qualidade de nado na definição das cargas de treino (Pelayo, Dekerle, Delaporte, Gosse, \& Sidney, 2000).

\section{Velocidade crítica anaeróbia - um conceito emergente}

Os problemas metodológicos relacionados com o cálculo da VC, associados à necessidade crescente de especialização do treino desportivo, desencadearam o desenvolvimento de novas abordagens e perspectivas de avaliação científica. Apesar da variedade de abordagens existentes, Fernandes, Aleixo, Soares, e Vilas-Boas (2008) chegaram a considerar que o cálculo da VC com recurso a distâncias mais curtas poderia ter forte relação com o uso dos sistemas bioenergéticos mais potentes. Com base nesta ideia, muito recentemente surgiu um conceito que tem despontado no seio da comunidade técnica e científica da natação que é o de velocidade crítica anaeróbia $\left(\mathrm{VC}_{\mathrm{an}}\right)$. A determinação da $\mathrm{VC}_{\mathrm{an}}$ regese pelo mesmo princípio em que se suporta o cálculo da VC, ou seja, pela obtenção do declive da recta de regressão, no entanto com base em distâncias mais curtas (Abe et al., 2006; Fernandes et al., 2008; Marinho et al., 2012; Neiva et al., 2011).

Na literatura são escassos os estudos que até ao momento se dedicaram a esta temática, objetivando para tal o cálculo da VC e tentando estabelecer a sua relação com o desempenho anaeróbio (tabela 2). Abe et al. (2006) recorreram às distancias de 75, 100 e 150 m para 
o cálculo da $\mathrm{VC}_{\text {an }}$ na técnica de bruços. Os autores observaram uma forte correlação $(r=0.85) \mathrm{da}$ $\mathrm{VC}_{\mathrm{an}}$ com o desempenho nos $50 \mathrm{~m}$ bruços. Uma abordagem semelhante foi efetuada por Fernandes et al. (2008) em nadadores jovens, contudo suportada na técnica de crol. Fortes relações foram observadas entre a $\mathrm{VC}_{a n}$ e as velocidades do primeiro $(r=0.73)$ e do segundo $(\mathrm{r}=0.60)$ parciais da prova de $100 \mathrm{~m}$ crol. Muito recentemente, Neiva, Fernandes, e Vilas-Boas (2011) tentaram descriminar o conceito de $\mathrm{VC}_{\mathrm{an}}$ baseando-se nas distâncias de $15,25,37.5$ e 50 nas quatro técnicas de nado. Como seria expectável os valores de $\mathrm{VC}_{\mathrm{an}}$ foram superiores a crol, mariposa, costas e bruços, respetivamente. Adicionalmente, foram observados fortes relações entre o desempenho nos $100 \mathrm{~m}$ e a $\mathrm{VC}_{\text {an }}$ de cada uma das técnicas de nado (mariposa $\mathrm{r}=$ 0.60 ; costas $r=0.81$; bruços $r=0.83$; crol $r=$ $0.78)$, com maior ênfase no primeiro parcial da prova. Marinho, Barbosa, Silva, e Neiva (2012) verificaram uma forte correlação entre a $\mathrm{VC}_{\mathrm{an}}$ (utilizando as distâncias de 10, 15, 20 e $25 \mathrm{~m}$ ) e o desempenho nos 50, 100 e $200 \mathrm{~m}$ crol em jovens nadadores de ambos os sexos. Para além disso, não foram encontradas diferenças entre a velocidade dos $200 \mathrm{~m}$ e a $\mathrm{VC}_{\mathrm{an}}$, sugerindo que a velocidade crítica anaeróbia pode ser um parâmetro importante para a monitorização dos efeitos do treino, especialmente para a prova de $200 \mathrm{~m}$ livres. Contudo não parece existir uma relação consistente entre $\mathrm{VC}_{\mathrm{an}} \mathrm{e}$ distância máxima de nado neste regime o que significa que nem sempre um elevado desempenho anaeróbio, traduzida em velocidade de nado, conduzirá a desempenhos desportivos elevados (Louro et al., 2013), sobretudo em distâncias de nado entre os 100 e 200m. Assim poderá ser importante considerar no futuro a distância máxima de nado a esta velocidade, como indicador de aptidão anaeróbia total do nadador.

Tabela 2.

Estudos centrados no cálculo da velocidade crítica anaeróbia em natação com base na combinação de coordenadas e distâncias de nado.

\begin{tabular}{|c|c|c|c|c|}
\hline \multirow[b]{2}{*}{ Autor } & \multicolumn{2}{|c|}{ Amostra } & \multirow[b]{2}{*}{$\begin{array}{l}\text { Distâncias de } \\
\text { nado }(\mathrm{m})\end{array}$} & \multirow[b]{2}{*}{ Resultados } \\
\hline & $\mathrm{N}$ & $\begin{array}{l}\text { Idade } \\
\text { (anos) }\end{array}$ & & \\
\hline Abe et al. (2006) & $\begin{array}{l}11 \text { nadadores, } \\
\text { treinados }\end{array}$ & $(19.7 \pm 0,9)$ & 75,100 e 150 & $\begin{array}{l}\text { Correlação }(\mathrm{r}=0.85) \text { da } \quad \mathrm{VC}_{\mathrm{an}} \text { com } \mathrm{o} \\
\text { desempenho nos } 50 \mathrm{~m} \text { bruços. }\end{array}$ \\
\hline Fernandes et al. (2008) & $\begin{array}{c}32 \text { nadadores, } \\
\text { treinados }\end{array}$ & (12 à 13) & $12,5,25$ e 50 & $\begin{array}{l}\text { Correlação inversa entre a } \mathrm{VC}_{\mathrm{an}} \text { e o tempo } \\
\text { dos } 100 \mathrm{~m} \text { crol }(\mathrm{r}=-0.84) \text { e as velocidades } \\
\text { do primeiro }(\mathrm{r}=-0.87) \text { e do segundo }(\mathrm{r}=- \\
0.79) \text { parciais da prova. }\end{array}$ \\
\hline Neiva et al. (2011) & $\begin{array}{l}51 \text { nadadores, } \\
\text { treinados }\end{array}$ & $\begin{array}{c}(20.2 \pm \\
3.25)\end{array}$ & $15,25,37,5$ e 50 & $\begin{array}{l}\text { Correlação entre o desempenho nos } 100 \mathrm{~m} \\
\text { e a } \mathrm{VC}_{\text {an }} \text { de cada uma das técnicas de nado } \\
\text { (mariposa, } \mathrm{r}=0,60 \text {; costas, } \mathrm{r}=0.81 \text {; bruços, } \\
\mathrm{r}=0.83 \text {; crol, } \mathrm{r}=0.78 \text { ) }\end{array}$ \\
\hline Marinho et al. (2012) & $\begin{array}{c}20 \text { nadadores, } \\
\text { treinados }\end{array}$ & (12 a 13) & $10,15,20$ e 25 & $\begin{array}{l}\text { Correlação entre a } \mathrm{VC}_{\text {an }} \text { e o desempenho } \\
\text { nos } 50(\mathrm{r}=0.85), 100 \quad(\mathrm{r}=0.91) \text { e } 200 \\
(\mathrm{r}=0.90) \text { crol. }\end{array}$ \\
\hline Louro et al. (2013) & $\begin{array}{l}9 \text { nadadores, } \\
\text { treinados }\end{array}$ & $(18.4 \pm 3.2)$ & 15,25 e 50 & $\begin{array}{l}\text { Correlação entre a } \mathrm{VC}_{\mathrm{an}} \text { e o desempenho } \\
\text { nos } 100 \mathrm{~m} \text { crol }(\mathrm{r}=0.91) \text {. A distância de } \\
\text { nado máxima à } \mathrm{VC}_{\mathrm{an}} \text { foi de } 97.22 \mathrm{~m} \\
( \pm 20.51) \text {, não correlacionado com a } \mathrm{VC}_{\mathrm{an}} \text {. }\end{array}$ \\
\hline
\end{tabular}

$\mathrm{VC}_{\mathrm{an}}$ - velocidade crítica anaeróbia

Em suma, até a este momento, e não existindo evidência científica em contrário, a $\mathrm{VC}_{\mathrm{an}}$ parece ser um indicador apropriado para controlar a intensidade de nado durante as séries de treino anaeróbio, especialmente se considerarmos neste âmbito as tarefas mais relacionadas com a capacidade anaeróbia. Embora o conceito de $\mathrm{VC}_{\text {an }}$ ainda seja um conceito vago, o uso de séries fracionadas em repetições até uma distância de
$50 \mathrm{~m}$ parece ser uma boa ferramenta para o desenvolvimento das características anaeróbias do nadador. Assim, a velocidade a ser adotada em cada parcial deverá ser previamente obtida pela relação distância-tempo usando as distâncias parcelares mais curtas como sejam os 10, 15, 20 e $25 \mathrm{~m}$. Neste sentido, a monitorização regular da $\mathrm{VC}_{\text {an }}$ poderá permitir um controlo mais efetivo do processo de treino, especialmente por este ser 
considerado um método não invasivo. Contudo, este é um conceito relativamente recente e não totalmente esclarecido, o que necessita de mais investigação.

\section{CONSIDERAÇÕES FINAIS}

A VC é sugerida como um parâmetro não invasivo de avaliação do desempenho aeróbio e de predição do limiar anaeróbio de nadadores, independentemente do nível desportivo. As principais vantagens deste indicador resultam da facilidade de aplicação e análise num elevado número de nadadores, sem a necessidade da utilização de equipamentos dispendiosos ou de qualquer recolha de sangue. Aponta-se como uma ferramenta útil no mecanismo de prescrição e controlo de cargas de treino por meio de resultados em condições experimentais ou recorrendo a tempos obtidos durante as competições. As diferentes combinações de distâncias e coordenadas propostas pela literatura afectaram a reprodutibilidade dos estudos e por consequência o significado fisiológico deste indicador, em particular na natação. Por isso, salienta-se a necessidade de aumentar a fiabilidade dos registos em intervenções futuras, tanto pelo uso das mesmas distâncias no cálculo da VC, como pela correção dos registos caso estes sejam obtidos em condições de nado distintas (piscina longa/curta; partida com/sem salto). A maioria dos estudos são suportados em metodologias estatísticas simples (por vezes não paramétricas), recorrendo a amostras de pequena dimensão, maioritariamente no género masculino e geralmente não descriminantes da especialidade desportiva. Assim, tal como apontam alguns estudos recentes, futuras abordagens deverão considerar abordagens estatísticas mais robustas e a descriminação dos valores de velocidade crítica entre nadadores com diferentes perfis metabólicos (velocistas versus fundistas). A opção pelo uso de modelos com 3 ou 4 parâmetros, recorrendo a mais distâncias de nado parece ser a mais ajustada para a prescrição dos ritmos de treino, embora isso pressuponha menor praticidade. De qualquer modo, os treinadores poderão sempre que possível recorrer aos tempos de prova para colmatar a necessidade de cálculo. Por último, urge aprofundar a aplicabilidade do conceito em regime de esforço anaeróbio, especialmente porque os estudos parecem apontar que a $\mathrm{VC}_{\text {an }}$ anaeróbia pode ser utilizada na prescrição e controlo da intensidade de nado em tarefas relacionadas com o desenvolvimento da capacidade anaeróbia.

\section{Agradecimentos: \\ Nada a declarar.}

Conflito de Interesses:

Nada a declarar.

\section{Financiamento:}

Nada a declarar

\section{REFERÊNCIAS}

Abe, D., Tokumaru, H., Niihata, S., Muraki, S., Fukuoka, Y., Usui, S., \& Yoshida, T. (2006). Assessment of short-distance breaststroke swimming performance with critical velocity. Journal of Sports Science \& Medicine, 5(2), 340-348.

Altimari, J. M., Altimari, L. R., Gulak, A., \& ChaconMikahil, M. P. T. (2007). Correlations between anaerobic threshold determination protocols and aerobic performance in adolescent swimmers. Revista Brasileira de Medicina do Esporte, 13(4), 245-250. http://doi.org/10.1590/S151786922007000400007

Barden, J. M., \& Kell, R. T. (2009). Relationships between stroke parameters and critical swimming speed in a sprint interval training set. Journal of Sports Sciences, 27(3), 227-235. http://doi.org/10.1080/02640410802475205

Berthoin, S., Baquet, G., Dupont, G., \& Van Praagh, E. (2006). Critical velocity during continuous and intermittent exercises in children. European Journal of Applied Physiology, 98(2), 132-138. http://doi.org/10.1007/s00421-006-0253-2

Billat, V. L., Sirvent, P., Py, G., Koralsztein, J.-P., \& Mercier, J. (2003). The concept of maximal lactate steady state: a bridge between biochemistry, physiology and sport science. Sports Medicine (Auckland, N.Z.), 33(6), 407-426. http://doi.org/10.2165/00007256-20033306000003

Brickley, G., Doust, J., \& Williams, C. A. (2002). Physiological responses during exercise to exhaustion at critical power. European Journal of Applied Physiology, 88(1-2), 146-151. http://doi.org/10.1007/s00421-002-0706-1 
Capelli, C., Pendergast, D. R., \& Termin, B. (1998). Energetics of swimming at maximal speeds in humans. European Journal of Applied Physiology and Occupational Physiology, 78(5), 385-393. http://doi.org/10.1007/s004210050435

Cardoso, C., Fernandes, R., \& Vilas-Boas, J. P. (2003). Comparison of continuous and intermittent incremental protocols for direct VO2max assessment. Em J. C. Chatard (Ed.), Biomechanics and Medicine in Swimming IX (pp. 313-318). SaintÉtienne: Publications de l'Université de SaintÉtienne.

Costa, A. M., Silva, A. J., Louro, H., Reis, V. M., Garrido, N. D., Marques, M. C., \& Marinho, D. A. (2009). Can the curriculum be used to estimate critical velocity in young competitive swimmers? Journal of Sports Science \& Medicine, 8(1), 1723.

Dekerle, J., Brickley, G., Sidney, M., \& Pelayo, P. (2006). Application of the critical power concept in swimming. Portuguese Journal of Sport Sciences, 6(S2), 103-105.

Dekerle, J., Pelayo, P., Clipet, B., Depretz, S., Lefevre, T., \& Sidney, M. (2005). Critical swimming speed does not represent the speed at maximal lactate steady state. International Journal of Sports Medicine, 26(7), 524-530. http://doi.org/10.1055/s-2004-821227

Dekerle, J., Sidney, M., Hespel, J. M., \& Pelayo, P. (2002). Validity and reliability of critical speed, critical stroke rate, and anaerobic capacity in relation to front crawl swimming performances. International Journal of Sports Medicine, 23(2), 93-98. http://doi.org/10.1055/s-2002-20125

Denadai, B. S., Greco, C. C., \& Teixeira, M. (2000). Blood lactate response and critical speed in swimmers aged 10-12 years of different standards. Journal of Sports Sciences, 18(10), 779-784. http://doi.org/10.1080/026404100419838

di Prampero, P. E. (1999). The concept of critical velocity: a brief analysis. European Journal of Applied Physiology and Occupational Physiology, $80(2)$, 162-164. http://doi.org/10.1007/s004210050574

di Prampero, P. E., Dekerle, J., Capelli, C., \& Zamparo, P. (2008). The critical velocity in swimming. European Journal of Applied Physiology, 102(2), 165-171. http://doi.org/10.1007/s00421-0070569-6

Ettema, J. H. (1966). Limits of human performance and energy-production. Internationale Zeitschrift Für Angewandte Physiologie Einschließlich Arbeitsphysiologie, 22(1), 45-54. http://doi.org/10.1007/BF00694796

Faina, M., Billat, V., Squadrone, R., De Angelis, M., Koralsztein, J. P., \& Dal Monte, A. (1997). Anaerobic contribution to the time to exhaustion at the minimal exercise intensity at which maximal oxygen uptake occurs in elite cyclists, kayakists and swimmers. European Journal of Applied Physiology and Occupational Physiology, 76(1), 13-20.
Fernandes, R. J., Aleixo, I., Soares, S., \& Vilas-Boas, J. P. (2008). Anaerobic Critical Velocity: A new tool for young swimmers training advice. Em W. Beaulieu (Ed.), Physical Activity and Children (pp. 211-223). New York: New Science Publishers.

Fernandes, R. J., Billat, V. L., Cruz, A. C., Colaço, P. J., Cardoso, C. S., \& Vilas-Boas, J. P. (2006). Does net energy cost of swimming affect time to exhaustion at the individual's maximal oxygen consumption velocity? The Journal of Sports Medicine and Physical Fitness, 46(3), 373-380.

Fernandes, R. J., Cardoso, C. S., Soares, S. M., Ascensão, A., Colaço, P. J., \& Vilas-Boas, J. P. (2003). Time limit and VO2 slow component at intensities corresponding to VO2max in swimmers. International Journal of Sports Medicine, 24(8), 576-581. http://doi.org/10.1055/s-200343274

Fernandes, R. J., Sousa, M., Machado, L., \& Vilas-Boas, J. P. (2011). Step length and individual anaerobic threshold assessment in swimming. International Journal of Sports Medicine, 32(12), 940946. http://doi.org/10.1055/s-0031-1283189

Fernandes, R. J., \& Vilas Boas, J. P. (1999). Critical velocity as a criterion for estimating aerobic training pace in juvenile swimmers. Em K. Keskinen, P. Komi, \& A. P. Hollander (Eds.), Biomechanics and medicine in swimming VIII (pp. 233-238). Jyvaskyla: Gummerus Printing.

Fernandes, R. J., \& Vilas-Boas, J. P. (2012). Time to Exhaustion at the VO2max Velocity in Swimming: A Review. Journal of Human Kinetics, 32, 121-134. http://doi.org/10.2478/v10078-0120029-1

Franken, M., Zacca, R., \& Castro, F. A. de S. (2010). Critical speed in swimming: theoretical basis and application. Motriz, 17(1), 209-222. http://doi.org/10.5016/19806574.2011v17n1p209

Gaesser, G. A., Carnevale, T. J., Garfinkel, A., Walter, D. O., \& Womack, C. J. (1995). Estimation of critical power with nonlinear and linear models. Medicine and Science in Sports and Exercise, 27(10), 1430-1438.

Gaesser, G. A., \& Poole, D. C. (1996). The slow component of oxygen uptake kinetics in humans. $E_{X}$ ercise and Sport Sciences Reviews, 24, 35-71.

Greco, C. C., \& Denadai, B. S. (2005). Critical speed and endurance capacity in young swimmers: effects of gender and age. Pediatric Exercice Science, 17(4), 353-363.

Greco, C. C., Denadai, B. S., Pellegrinotti, I. L., Freitas, A. B., \& Gomide, E. (2003). Limiar anaeróbio e velocidade crítica determinada com diferentes distâncias em nadadores de 10 a 15 anos: relações com a performance e a resposta do lactato sanguíneo em testes de endurance. Revista Brasileira de Medicina do Esporte, 9(1), 2-8. http://doi.org/10.1590/S151786922003000100002 
Heck, H., Mader, A., Hess, G., Mücke, S., Müller, R., \& Hollmann, W. (1985). Justification of the 4$\mathrm{mmol} / \mathrm{l}$ lactate threshold. International Journal of Sports Medicine, 6(3), 117-130. http://doi.org/10.1055/s-2008-1025824

Hill, D. W. (1993). The critical power concept. A review. Sports Medicine (Auckland, N.Z.), 16(4), $237-254$.

Hill, D. W. (2001). Aerobic and anaerobic contribution in middle distance running events. Motriz, $7(\mathrm{~S} 1)$, 63-67.

Hill, D. W., \& Ferguson, C. S. (1999). A physiological description of critical velocity. European Journal of Applied Physiology and Occupational Physiology, 79(3), 290-293. http://doi.org/10.1007/s004210050509

Hill, D. W., \& Smith, J. C. (1999). Determination of critical power by pulmonary gas exchange. Canadian Journal of Applied Physiology = Revue Canadienne De Physiologie Appliquée, 24(1), 74-86.

Hill, D. W., Steward Jar, R. P., \& Lane, C. J. (1995). Application of the critical power concept to young swimmers. Pediatric Exercice Science, 7(3), 281-293.

Hinckson, E. A., \& Hopkins, W. G. (2005). Reliability of time to exhaustion analyzed with criticalpower and log-log modeling. Medicine and Science in Sports and Exercise, 37(4), 696-701.

Housh, T. J., Devries, H. A., Housh, D. J., Tichy, M. W., Smyth, K. D., \& Tichy, A. M. (1991). The relationship between critical power and the onset of blood lactate accumulation. The Journal of Sports Medicine and Physical Fitness, 31(1), 3136.

Jones, A. M., \& Poole, D. C. (2005). Oxygen Uptake Kinetics in Sport, Exercise and Medicine. London: Routledge.

Keskinen, K. L., \& Komi, P. V. (1993). Stroking characteristics of front crawl swimming during exercise. Journal Applied Biomechanics, 9(3), 219226.

Louro, H., Silva, P., Conceição, A. T., Neiva, H., Marinho, D. A., \& Costa, A. M. (2013). Maximal swimming distance at anaerobic critical velocity. International Journal of Swimming Kinetics, 2(1), 71-86.

Mader, A., \& Heck, H. (1986). A theory of the metabolic origin of «anaerobic threshold». International Journal of Sports Medicine, 7(S1), 45-65.

Marinho, D. A., Barbosa, T. M., Silva, A. J., \& Neiva, H. P. (2012). Applying Anaerobic Critical Velocity in Non-Elite Swimmers. International Journal of Swimming Kinetics, 1(1), 33-50.

Martin, L., \& Whyte, G. P. (2000). Comparison of critical swimming velocity and velocity at lactate threshold in elite triathletes. International Journal of Sports Medicine, 21(5), 366-368. http://doi.org/10.1055/s-2000-3786

Monod, H., \& Scherrer, J. (1965). The work capacity of a synergic muscular group. Ergonomics, 8(3),
$329-338$.

http://doi.org/10.1080/00140136508930810

Moritani, T., Nagata, A., deVries, H. A., \& Muro, M. (1981). Critical power as a measure of physical work capacity and anaerobic threshold. Ergonomics, 24(5), 339-350. http://doi.org/10.1080/00140138108924856

Morton, R. H. (1996). A 3-parameter critical power model. Ergonomics, 39(4), 611-619. http://doi.org/10.1080/00140139608964484

Morton, R. H. (2006). The critical power and related whole-body bioenergetic models. European Journal of Applied Physiology, 96(4), 339-354. http://doi.org/10.1007/s00421-005-0088-2

Neiva, H. P., Fernandes, R. J., \& Vilas-Boas, J. P. (2011). Anaerobic critical velocity in four swimming techniques. International Journal of Sports Medicine, 32(3), 195-198. http://doi.org/10.1055/s-0030-1268474

Papoti, M., Zagatto, A. M., Mendes, O. C., \& Gobatto, C. A. (2005). Utilização de métodos invasivo e não invasivo na predição das performances aeróbia e anaeróbia em nadadores de nível nacional. Revista Portuguesa de Ciências do Desporto, 5(1), 07-14.

Pelayo, P., Dekerle, J., Delaporte, B., Gosse, N., \& Sidney, M. (2000). Critical speed and critical stroke rate could be useful physiological and technical criteria for coaches to monitor endurance performance in competitive swimmers. Em abstract book XVIII International Symposium on Biomechanics in Sports. Hong Kong.

Poole, D. C., Ward, S. A., Gardner, G. W., \& Whipp, B. J. (1988). Metabolic and respiratory profile of the upper limit for prolonged exercise in man. Ergonomics, $31(9)$, 1265-1279. http://doi.org/10.1080/00140138808966766

Poole, D. C., Ward, S. A., \& Whipp, B. J. (1990). The effects of training on the metabolic and respiratory profile of high-intensity cycle ergometer exercise. European Journal of Applied Physiology and Occupational Physiology, 59(6), 421-429.

Reis, V. M., Marinho, D. A., Policarpo, F. B., Carneiro, A. C., Baldari, C., \& Silva, A. J. (2010). Examining the accumulated oxygen deficit method in front crawl swimming. International Journal of Sports Medicine, 31(6), 421-427. http://doi.org/10.1055/s-0030-1248286

Reis, V. M., Marinho, D. A., Policarpo, F. B., Reis, A. M., Guidetti, L., \& Silva, A. J. (2010). Examining the accumulated oxygen deficit method in breaststroke swimming. European Journal of Applied Physiology, 109(6), 1129-1135. http://doi.org/10.1007/s00421-010-1460-4

Ribeiro, L., Balikian, P., Malachias, P., \& Baldissera, V. (2003). Stage length, spline function and lactate minimum swimming speed. The Journal of Sports Medicine and Physical Fitness, 43(3), 312-318.

Rodriguez, F. A., Moreno, D., \& Keskinen, K. L. (2003). Validity of a two-distance simplified testing method for determining critical swimming 
velocity. Em Biomechanics and Medicine in Swimming IX (Publications de l'Université de Saint-Étienne, pp. 385-390). Saint-Etienne: Publications de l'Université de Saint-Étienne.

Smith, J. C., \& Hill, D. W. (1993). Stability of parameter estimates derived from the power/time relationship. Canadian Journal of Applied Physiology = Revue Canadienne De Physiologie Appliquée, 18(1), 43-47.

Spencer, M. R., \& Gastin, P. B. (2001). Energy system contribution during 200- to $1500-\mathrm{m}$ running in highly trained athletes. Medicine and Science in Sports and Exercise, 33(1), 157-162.

Stegmann, H., Kindermann, W., \& Schnabel, A. (1981). Lactate kinetics and individual anaerobic threshold. International Journal of Sports Medicine, 2(3), 160-165. http://doi.org/10.1055/s2008-1034604

Tegtbur, U., Busse, M. W., \& Braumann, K. M. (1993). Estimation of an individual equilibrium between lactate production and catabolism during exercise. Medicine and Science in Sports and Exercise, 25(5), 620-627.

Toubekis, A. G., Tsami, A. P., Smilios, I. G., Douda, H. T., \& Tokmakidis, S. P. (2011). Training-induced changes on blood lactate profile and critical velocity in young swimmers. Journal of Strength and Conditioning Research, 25(6), 1563-1570. http://doi.org/10.1519/JSC.0b013e3181ddfafc

Vandewalle, H., Kapitaniak, B., Grün, S., Raveneau, S., \& Monod, H. (1989). Comparison between a 30$\mathrm{s}$ all-out test and a time-work test on a cycle ergometer. European Journal of Applied Physiology and Occupational Physiology, 58(4), 375-381.

Vilas-Boas, J. P., Lamares, J. P., Fernandes, R., \& Duarte, J. A. (1997). Avaliação do nadador e definição de objetivos através de critérios não invasivos de simples implementação. Horizonte: Revista de Educação Física e Desporto, 14(80), 22-30.

Wakayoshi, K., Ikuta, K., Yoshida, T., Udo, M., Moritani, T., Mutoh, Y., \& Miyashita, M. (1992). Determination and validity of critical velocity as an index of swimming performance in the competitive swimmer. European Journal of Applied Physiology and Occupational Physiology, 64(2), 153157.
Wakayoshi, K., Yoshida, T., Udo, M., Harada, T., Moritani, T., Mutoh, Y., \& Miyashita, M. (1993). Does critical swimming velocity represent exercise intensity at maximal lactate steady state? European Journal of Applied Physiology and Occupational Physiology, 66(1), 90-95.

Wakayoshi, K., Yoshida, T., Udo, M., Kasai, T., Moritani, T., Mutoh, Y., \& Miyashita, M. (1992). A simple method for determining critical speed as swimming fatigue threshold in competitive swimming. International Journal of Sports Medicine, 13(5), 367-371. http://doi.org/10.1055/s2007-1021282

Whipp, B. J., \& Wasserman, K. (1972). Oxygen uptake kinetics for various intensities of constant-load work. Journal of Applied Physiology, 33(3), 351356.

Wilkie, D. R. (1980). Equations describing power input by humans as a function of duration of exercise. Em P. Cerretelli \& B. J. Whipp (Eds.), Exercise bioenergetics and gas exchange (pp. 75-80). Amsterdam: Elsevier.

Wright, B., \& Smith, D. J. (1994). A protocol for the determination of critical speed as an index of swimming endurance performance. Em M. Miyashita, Y. Mutoh, \& A. B. Richardson (Eds.), Medicine and Science in Aquatic Sports (pp. 5559). Basel: Karger.

Zacca, R., \& Castro, F. A. (2009). Comparação entre diferentes modelos de obtenção de velocidade crítica em nadadores juvenis. Revista Brasileira de Fisiologia do Exercício, 8(2), 52-60.

Zacca, R., Wenzel, B. M., Piccin, J. S., Marcilio, N. R., Lopes, A. L., \& Castro, F. A. (2010). Critical velocity, anaerobic distance capacity, maximal instantaneous velocity and aerobic inertia in sprint and endurance young swimmers. European Journal of Applied Physiology, 110(1), 121-131. http://doi.org/10.1007/s00421-010-1479-6

Zamparo, P., Capelli, C., \& Pendergast, D. (2011). Energetics of swimming: a historical perspective. European Journal of Applied Physiology, 111(3), 367-378. http://doi.org/10.1007/s00421-0101433-7 